\title{
INFORMATION RELIABILITY ENHANCEMENT METHODS
}

Salmanov V.I.

Nakhchivan State University

\begin{abstract}
Information reliability is the property of information to reflect real-world objects with the necessary accuracy. The reliability of information is an essential characteristic of any communication system.

It is found that in modern network architectures of test automation systems, the main problem in the field of enhancement of the reliability of information is the creation of methods aimed at solving the problem of enhancing the reliability for "real" time systems.

Keywords: information, reliability of information, private channel, key
\end{abstract}

The most widely used architecture of test automation systems for complex objects implemented in the class of information and control systems is a network architecture with a vertical hierarchy of implemented functions [1]. Let's review a generalized level model of the test automation system (Figure 1).

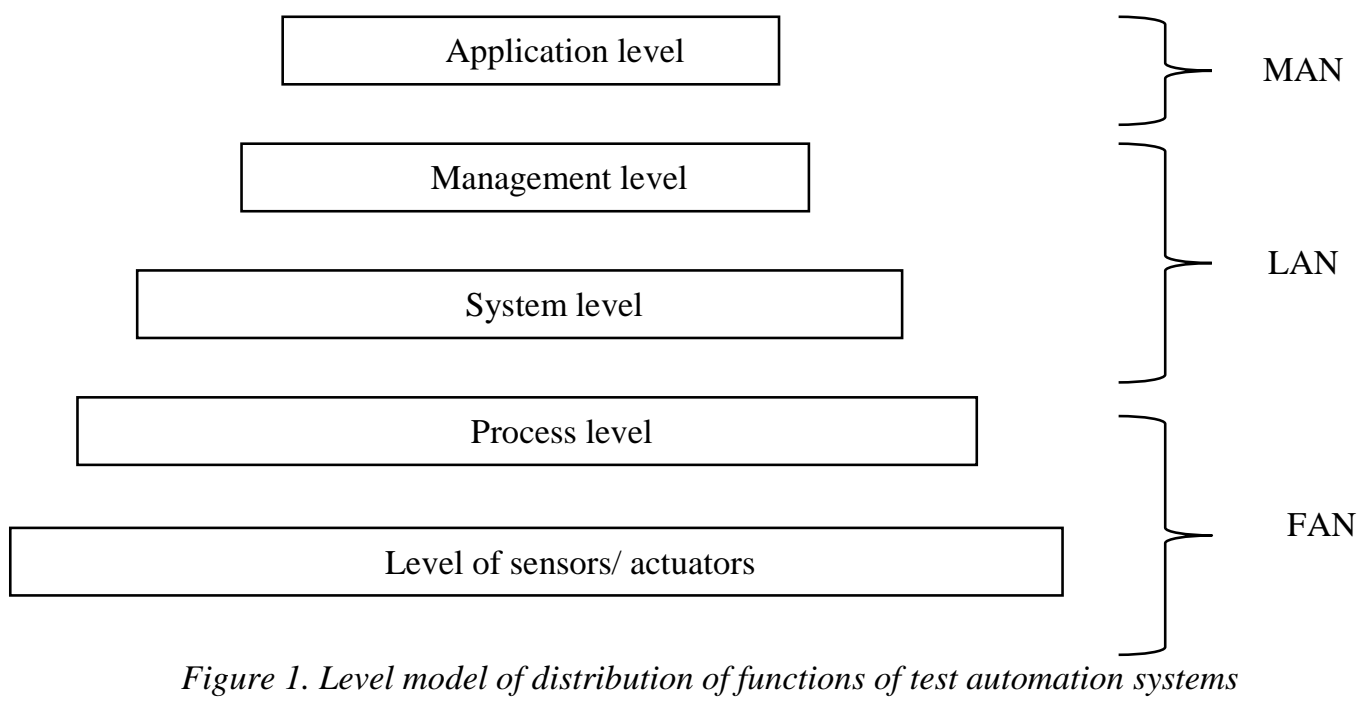

The distribution of functional tasks by levels is determined by specific types and test programs and it significantly depends on the set of parameters of the test object. The integration of levels into the system is implemented using network protocols. So, FAN (Fieldbus Area Networks) protocols are used at level 1 and 2 [2], LAN (Local Area Networks) protocols are used at levels 3 and 4 [2], and MAN (Metropoliten Area Networks) protocols are used at level 5 [2].

Let's note some features of the architecture under consideration.

1. Autonomy and functional completeness of each level. Each of the levels considered is able to perform tasks completely independently, perform control actions, interact with other levels, receive and issue messages and data. All these actions are performed independently of other levels, through connecting to the switching network only upon exchanging operations.

2. Distribution of hardware and software between the system levels. All technical tools of the levels are independent, and a hierarchical distributed network operating system controls the solution of tasks and the functioning of equipment facilities.

3. The versatility of the communication network. The AIS flexibility and adaptability is sufficiently specified by the versatility of network protocols.

4. Distribution of real-time and scalable time modes.

It is necessary to enhance the reliability of information in test automation systems through the use of special coding methods at all levels of the system. However, the currently existing coding methods are mainly focused on adoption in MAN and LAN type structures [2]. Therefore, the development and creation of methods and algorithms aimed at enhancing the reliability of "real" time systems operating on the basis of FAN is relevant, and the research in this paper shall be aimed at solving such a problem.

Let's review the existing methods for detecting and correcting errors that occur in the information. 
Let's consider the known methods to enhance the reliability of information in test automation systems, taking into account the features of such systems.

To enhance the reliability upon information communication procedures, the following several groups of methods are used [3]:

1) the methods based on the repetition of the communication of a symbol or message, followed by a comparison of the texts received;

2) the methods involving the use of redundant coding operations;

3) the methods based on the data communication with the application of the feedback;

4) the methods providing for the use of special coding tools.

Taking into account the disadvantages of the methods above, we shall further focus on the analysis, construction and evaluation of special encoding methods that provide the information with the properties of non-concealability and security, taking into account the features of information communication, processing and transformation systems operating in "real" time mode.

To describe the network interaction, the International Organization for Standardization ISO (International Organization for Standardization) developed a model of computer network integration, which was called as OSI (Open System Interconnection), in Russian terminology it is a model of interaction of open systems. There is also a full name the ISO/OSI model [2]. The principle that underlies the model is quite simple. All the necessary communication functions are collected and arranged within a seven-level hierarchical system.

In the course of designing the ISO/OSI standard, the developers thought the least about the "real" time functions and did not foresee the occurrence of information protection problems. Despite this, later the ISO model was considered suitable for the specification and description of network communications.

All physical and mechanical parameters of the model are formed at the lower level, which is the determining one. At higher levels, the bus access method is implemented, the compilation of data frames is described and their protection is carried out during communication procedures (point-to-point communication of subscribers). At level 3, routing is performed, that is, the path selection. Level 4 controls the point-to-point communication: it is responsible for ensuring that the data packets sent by the transmitter reach the recipient in the right manner, that is, it controls the flow of information. At level 5, sessions are arranged - simultaneous data exchange between different subscribers. In certain situations, the tasks of this level are to identify participants and synchronize sessions after they are interrupted. At level 6, a common set of characters (language) is agreed upon. Level 7, like level 1, occupies a special place: it is an interface with the outside world.

In the ISO/OSI standard, enhancing the reliability of information through the use of coding methods is mainly focused on structures such as MAN and LAN [2].

Thus, it is not possible to use standard solutions of a seven-level data encoding model that provide an enhancement in the reliability of information communication in real-time modes.

The achievement of the properties of non-concealability and security of information may be ensured by using a secret key encoding method [3].

Let's consider the data communication process in the test automation system (see Figure 2). The data is communicated from point A to point B. A encodes (encodes) messages before communication, and C decodes them after they are received.

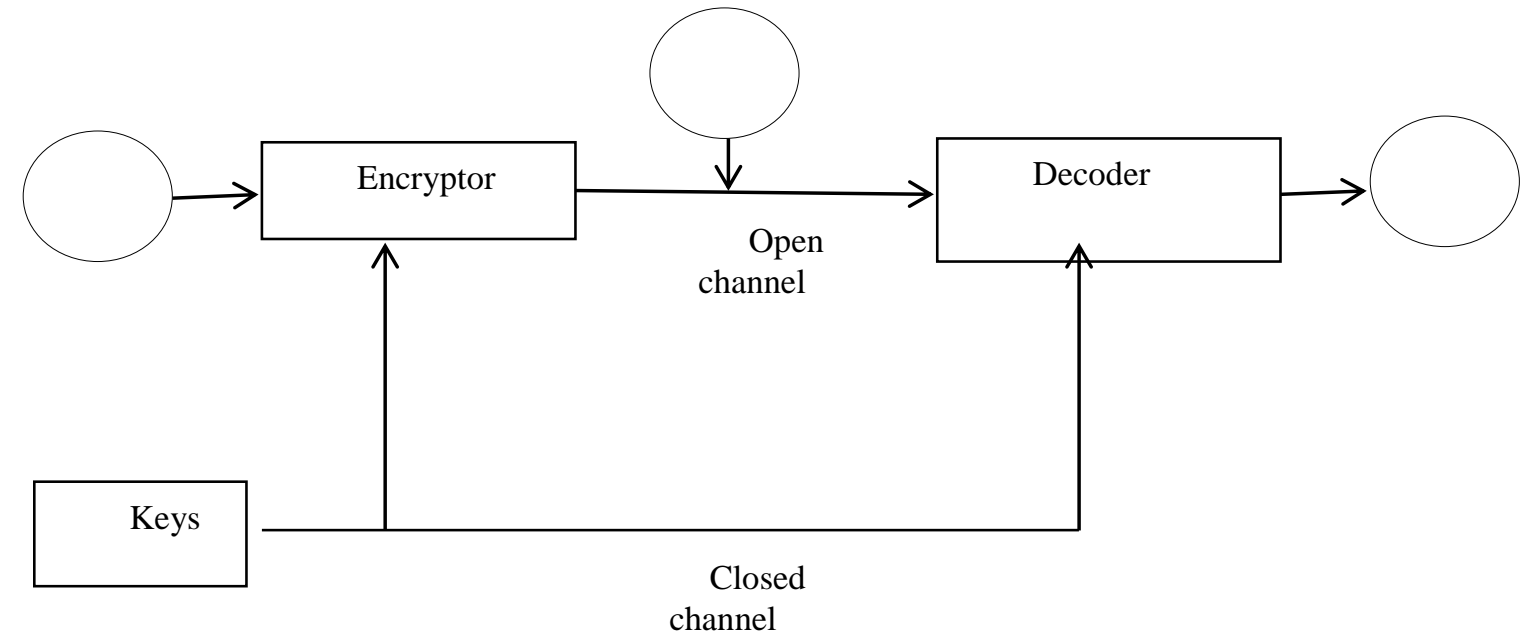

Figure 2. Data transmission system with a secret key 
Currently, block and stream codes are used for encoding data in such systems [4], which are the samples of the class of so-called symmetric encoding schemes or codes with a secret key [5].

It is important that A and B are to use the secret key not through an open channel, but through a special "closed" channel that is inaccessible to third parties or facilities. However, it should be considered in mind that usually the organization of such a closed channel and the transmission of messages through it are too expensive compared to an open channel and (or) a closed channel may not be used at any time.

It should be noted that currently a private channel or, more precisely, a secure channel may be created by means of cryptography with public keys.

Most of the data encoding methods that are practically used at the present time, upon transmitting information in "real" time systems, have only computational stability [5], i.e. the codes may be opened by brute force, and the absence of faster opening algorithms is not strictly proven. So, the following may be told really. The code is considered stable if it does not know hacking algorithms that are significantly more effective than direct key brute force.

The main approach used to build absolutely stable coding methods is based on using a random number generator as a key with a perfect random sequence of random number generation, the absence of any regularities in the generation sequence and an infinite gamming period. The method of building a random number generator itself should be open to any third parties.

Creating such a random number generator presents certain difficulties.

Therefore, a method for constructing a random number generator with the specified properties may be proposed, namely:

- $\quad$ there is no repetition period (gamming);

- $\quad$ the generated series of numbers is not subject to statistics;

- $\quad$ the simplicity of software implementation;

- high performance.

\section{References}

1. Aho A., Hopcroft J., Ullman J. Construction and analysis of computational algorithms. - M.: Mir, 1979. - p. 383.

2. Dietrich D., Loy D., Schweinzer G. Yu. LON-technology. Construction of distributed applications. // Perm: Zvezda.- 1999. - p. 424.

3. Volkova V. N., Kuzina B. M. Information systems. // Saint Petersburg: SPbSTU. - 1998. - p. 111.

4. Babkin V. F. Method of universal encoding of independent messages of non-exponential complexity.// Information transmission problems. - 1971.-V . 7,№4.-p. 13-21.

5.Introduction to Cryptography/ Under the general editorship by V.V.Yashchenko-M.: ICNMO: "CheRo", 2000. - p. 287. 